\title{
The successful non-invasive management of pulmonary thromboembolism in a child with acute lymphoblastic leukemia
}

\author{
Ikbal Ok Bozkaya, M.D. ${ }^{a}$, Ilker Cetin, M.D. ${ }^{b}$, Nese Yarali, M.D. ${ }^{a}$, Cengiz Bayram, M.D. ${ }^{a}$, Kanat Ozisik, M.D. ${ }^{c}$, \\ Mehmet E. Ari, M.D. ${ }^{b}$, and Bahattin Tunc, M.D. ${ }^{a}$
}

\begin{abstract}
In childhood, pulmonary thromboembolism (PTE) is an uncommon but potentially life-threatening disease. The greater numbers of venous thromboembolism (VTE) are complications of underlying risk factors such as malignancies, chemotherapy (L-asparaginase), and central venous catheter. We report a patient with acute lymphoblastic leukemia and PTE, who presented with near-syncope, and was successfully treated with low molecular weight heparin and calcium channel blockers. Key words: pulmonary embolism, leukemia, children.
\end{abstract}

http:/ /dx.doi.org/10.5546/aap.2016.eng.e17

\section{INTRODUCTION}

Pulmonary thromboembolism (PTE) is an obstruction of the main artery of the lung or one of its branches by a thrombus. In childhood, PTE is an uncommon but potentially life-threatening disease. Thromboembolism registry data has reported the annual incidence of childhood PTE as $0.14-0.9$ per 100,000 children. ${ }^{1,2}$ Risk factors of venous thromboembolism (VTE) are central venous catheter, malignancies, chemotherapy (L-asparaginase), congenital heart disease, infections, surgery, trauma, immobility, hypovolemia, lupus erythematosus, hemoglobinopathies (thalassemia, sickle cell disease), and inherited thrombotic disorders.

The diagnosis of PTE is difficult on clinical signs and pulmonary computed tomography

a. Department of Pediatric Hematology

b. Department of Pediatric Cardiology.

Ankara Children's Hematology Oncology Hospital.

c. Department of Cardiovascular Surgery, Yildirim Beyazit Training and Research Hospital.

Turkiye.

E-mail Address:

Ikbal Ok Bozkaya, M.D.: ikbalok@yahoo.com

Funding: None.

Conflict of interest: None.

Received: 7-8-2015

Accepted: 8-3-2015 angiography is the most widely used method in diagnosing PTE. ${ }^{3,4}$ Anticoagulation is the mainstay of therapy for PTE.

Pulmonary arterial hypertension (PAH) is defined as a mean pulmonary artery pressure (PAP) of more than $25 \mathrm{mmHg}$ at rest. PAH is rare in childhood and is a complex disorder with various etiologies. ${ }^{5}$

We report a case of PTE because of venous thrombosis at left brachial system, successfully and non-invasively managed in a teenage boy with leukemia.

\section{CASE REPORT}

A 16-year-old male patient presented with persistent leg pain and swelling of the lymph nodes in the neck which did not resolve with antimicrobial treatment. On physical examination he had bilateral sub-mandibular lymphadenopathy, hepatomegaly extending $5-6 \mathrm{~cm}$ below the right costal margin and splenomegaly extending $6-7 \mathrm{~cm}$ below the left costal margin. Additionally he had petechial rashes on his face and all extremities. Laboratory test results were as follows; hemoglobin: $9.1 \mathrm{~g} /$ dl; leukocyte: $258 \times 10^{9} / \mathrm{L}$; platelet: $36 \times 10^{9} / \mathrm{L}$; potassium: $5.7 \mathrm{mEq} / \mathrm{L}$; aspartate aminotransferase (AST): $103 \mathrm{IU} / \mathrm{L}$; alanine aminotransferase (ALT): $47 \mathrm{IU} / \mathrm{L}$; uric acid: $11.6 \mathrm{mg} / \mathrm{dl}$ (N: 2.40-7.00); lactate dehydrogenase: 4569 IU/L (N: 266.0500.0).

Lymphoblasts were the predominant cells in the peripheral blood smear. Flow cytometryc analysis revealed CD45, CD117, CD5, CD7, intracytoplasmic CD3 and CD22 positivity and the patient diagnosed as myeloid antigen-positive acute lymphoblastic leukemia (ALL) and TR-ALL BFM 2000 treatment protocol was started.

Since leukocyte count raised as high as 408 $\mathrm{x} 10^{9} / \mathrm{L}$ leukopheresis using left jugular central venous line was performed. Crystallized insulin due to secondary hyperglycemia following steroid use and teicoplanin, meropenem and fluconazole therapy for febrile neutropenia was administered. On day $24^{\text {th }}$, the catheter was removed without 
any complication. At second month of treatment, when there was a peripheral vascular catheter at left antecubital vein, hyperemia and pain appeared on the medial part of the left upper extremity. Doppler ultrasonography revealed increased intraluminal echogenity, increased vascular calibration and weak flow patern at left internal jugular vein, brachiocephalic junction, subclavian, axillary, brachial and basalic veins.

These findings were thought as the signs of recanalized thrombosis at subacute stage. Although, enoxaparin $100 \mathrm{U} / \mathrm{kg} /$ dose q12h was initiated, the patient developed a near-syncope episode lasting for 1-2 seconds after the second dose. Respiratory rate was $24 / \mathrm{min}$; heart rate was $120 / \mathrm{min}$, blood pressure was $100 / 70 \mathrm{mmHg}$ and oxygen saturation was $95 \%$. Echocardiographic examination was performed for evaluation for near-syncope and right ventricular dilation, moderate tricuspid valve insufficiency $(3.5 \mathrm{~m} / \mathrm{s}$, estimated systolic right ventricle pressure was 54 $\mathrm{mmHg}$ ), mild pulmonary valve insufficiency (2.6 $\mathrm{m} / \mathrm{s}$, estimated mean PAP was $27 \mathrm{mmHg}$ ) and as a result mild pulmonary hypertension was detected. Nasal oxygen (2 L/min), furosemid (1 $\mathrm{mg} / \mathrm{kg} /$ day, $1 \mathrm{x}, \mathrm{PO})$ and amlodipin $(0.1 \mathrm{mg} / \mathrm{kg} /$ day, $1 \mathrm{x}, \mathrm{PO}$ ) were started as well as enoxaparin. Because echocardiographic assessment revealed $27 \mathrm{mmHg}$ mean PAP, which was previously documented to be in the normal range, PTE was suspected.

The ventilation/perfusion scintigraphy and also computerized tomographic angiography

FIGURE 1. Thrombi with partial flow at right upper lobar artery

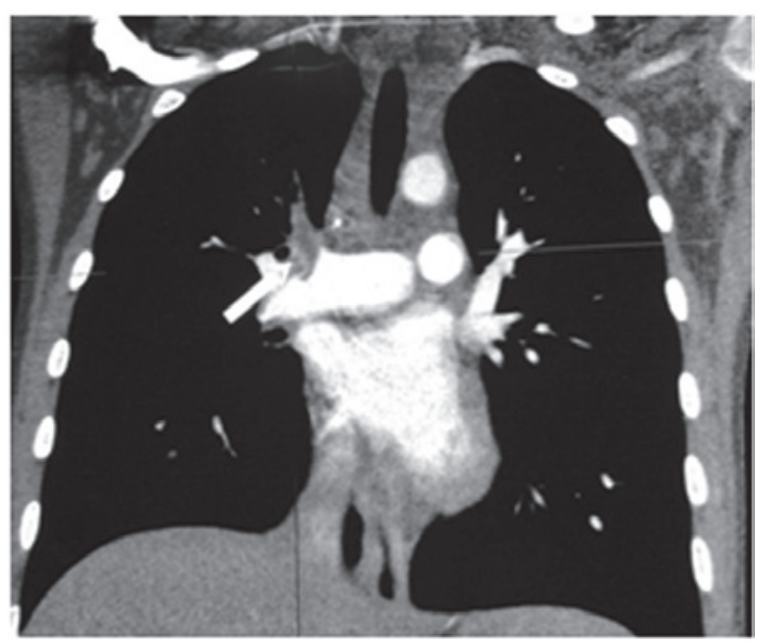

(CTA) of the pulmonary vascular bed revealed bilateral thrombi with partial flow at all lobar arteries of right main pulmonary artery and at all segmental arteries of left lower lobar artery (Figures 1 and 2). The dosing of enoxaparin was adjusted so as to keep anti-factor Xa level between 0.5-1.0. We did not undertake embolectomy because of the hemodynamic stability and good oxygenation of the patient. Protein $C$ and $S$, factor $\mathrm{V}$ Leiden, prothrombin gene mutation 20210 A, antithrombin III, homocysteine and antiphospholipid antibody levels were all in the normal range at that time.

Following medical treatment PAH gradually resolved (Figure 3), the patient achieved bone marrow remission and was discharged after the last TR ALL BFM 2000 High Risk consolidation therapy. Unfortunately, patient died because of neutropenic sepsis 5 months later from the diagnosis of ALL.

\section{DISCUSSION}

Pulmonary thromboembolism is a severe disorder with substantial mortality, especially when the diagnosis or treatment is delayed. ${ }^{4}$ Certain risk factors such as central venous catheterization, infection and congenital heart disease may increase the risk of PTE in children. The incidence of PTE in children with central venous catheters has been reported to be between 33 to $64 \% .4,6$

Previous studies reported that around $2.9 \%$ of children with malignancies sustained

FIGURE 2. Thrombi with partial flow at right lower lobar artery and segmental arteries of left lower lobar artery

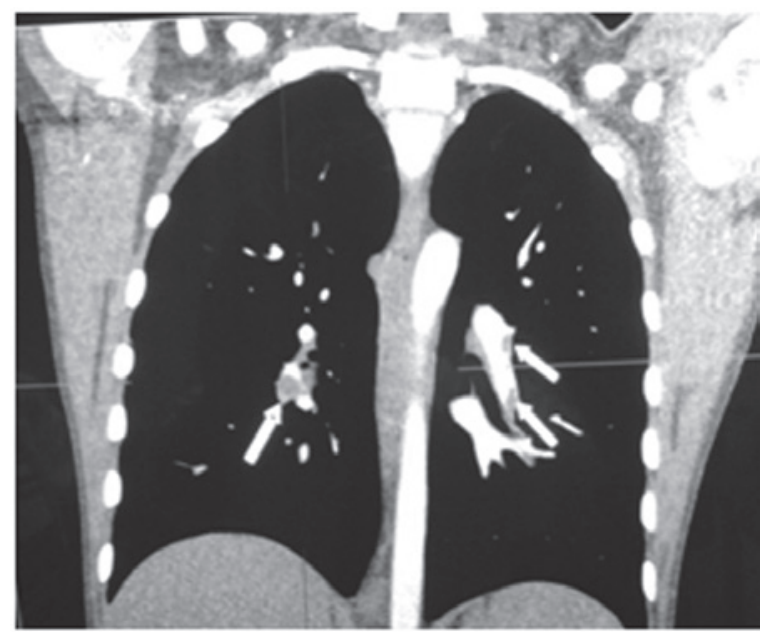


symptomatic PTE. The etiology of PTE in these children is multi-factorial and involves the use of central catheters, disorders of the coagulation system and disruption of the endothelial wall. ${ }^{7}$ Our patient had several risk factors including receiving leukophoresis due to elevated leukocytes, the central venous catheter, extensive chemotherapy exposure and steroid-induced hyperglycemia.

Shortness of breath, chest pain of a pleuritic nature, cough, hypoxemia, hemoptysis, tachycardia, fever and syncope are among the most common symptoms. ${ }^{8,9}$ More severe cases may present with right-sided heart failure symptoms and even cardiopulmonary arrest. Differential diagnosis should include pneumonia, atelectasis, intrathoracic malignity and trauma. ${ }^{4}$ Any episode of near-syncope in children with malignancies should raise suspicion of PTE. Echocardiography, performed following an episode of near-syncope in our patient, revealed PAH suggestive of PTE.

Suspicion of PTE should encourage the use of advanced diagnostic tools, even if initial blood gas analysis, electrocardiogram and other routine laboratory analyses revealed normal results. Echocardiography, ventilation/perfusion scintigraphy, CTA and pulmonary angiography are the primary diagnostic imaging modalities in PTE. ${ }^{10}$ Children, however, may not be suitable candidates for angiography due to the challenges in inserting a peripheral arterial line. ${ }^{6,7}$
Echocardiography may demonstrate directly the thrombus within the heart or the main pulmonary artery. Additionally it may reflect several indirect findings associated with embolism such as right ventricle dilatation, hypokinesia or abnormal inter-ventricular septum motion, tricuspid valve regurgitation and absence of the inferior vena cava collapse during inspiration. ${ }^{11}$ Following the observation of several indirect findings of PTE in echocardiography we performed V/Q and CTA to confirm the diagnosis.

In a series of 405 children with VTE the rate of mortality was $16 \%$ and $2.2 \%$ of which were due to VTE. Of the nine patients who died due to VTE, seven were of pulmonary origin and had a documented thrombus in the upper extremity as well as a central venous catheter. ${ }^{12}$ Embolectomy is rarely performed in children with a venous thrombosis and it is indicated merely in hemodynamically unstable children in whom thrombolysis fail or is contraindicated.

A single massive PE or repeated pulmonary emboli can lead to right ventricular failure, shock, and sudden death. Pulmonary arterial hypertension secondary to PE is considered a relatively rare complication. Various management strategies such as supportive therapy and lifestyle changes have been implemented for patients with PAH. Supportive therapy involves oral anticoagulant treatment, supplemental oxygen together with diuretic and digoxin therapy in right-heart failure. Calcium channel blockers

FIGURE 3. Echocardiographic mean pulmonary artery pressure (PAP) and right ventricular end diastolic diameter (RVEDD) measurements

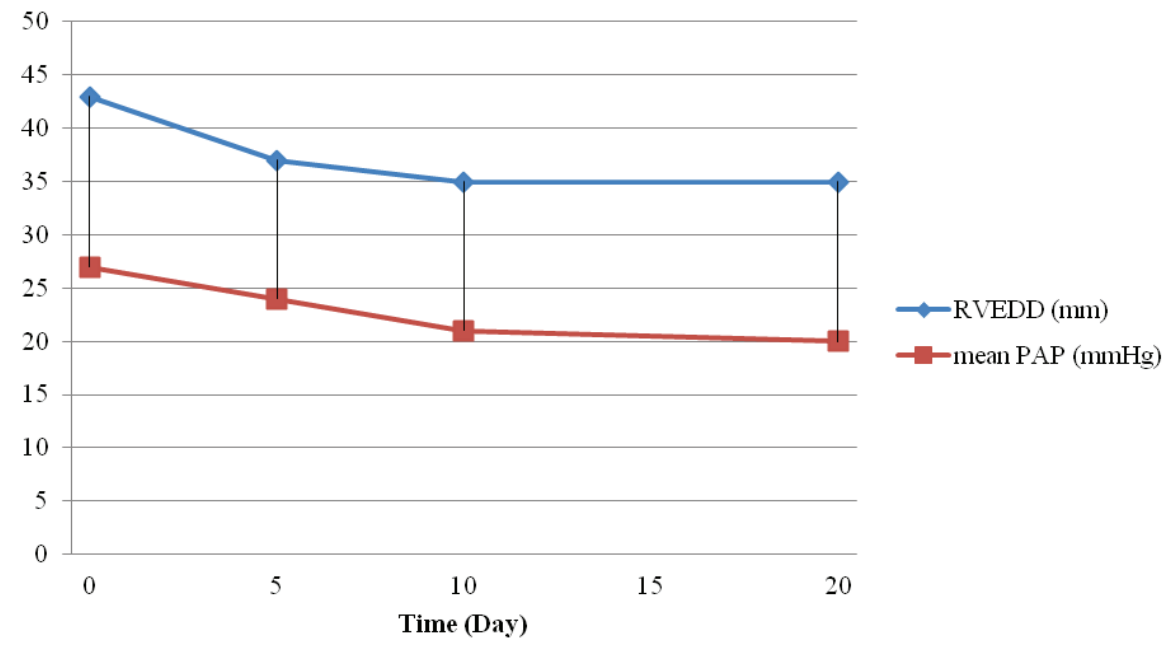


inhibit calcium influx into smooth muscle cells across the slow motion channels and result in vasodilatation of the smooth muscles of pulmonary vasculature..$^{10}$ Studies have shown that calcium channel blockers proved efficient in up to $40 \%$ of the children with PAH. ${ }^{6}$ Our patient was hemodynamically stabile and was prescribed low molecular weight heparin as an anticoagulant, a calcium channel blocker for PAH and a diuretic and digoxin for right-heart failure. His clinical status improved and pulmonary hypertension resolved gradually.

In the current report, we aimed to demonstrate that patients with hematologic malignancies are predisposed to thrombosis and that these patients required repetitive echocardiographic assessment to closely monitor the risk for pulmonary embolism.

\section{REFERENCES}

1. van Ommen $\mathrm{CH}$, Heijboer $\mathrm{H}$, Büller HR, Hirasing RA, et al. Venous thromboembolism in childhood: a prospective two-year registry in The Netherlands. JPediatr 2001;139(5):676-81.
2. Stein PD, Kayali F, Olson RE. Incidence of venous thromboembolism in infants and children: data from the National Hospital Discharge Survey. J Pediatr 2004;145(4):563-5.

3. van Ommen $\mathrm{CH}$, Peters $\mathrm{M}$. Venous thromboembolic disease in childhood. Semin Thromb Hemost 2003;29(4):391-404.

4. Dijk FN, Curtin J, Lord D, Fitzgerald DA. Pulmonary embolismin children. Paediatr Respir Rev 2012;13(2):112-22.

5. De Wolf D. Clinical practice: pulmonary hypertension in children. Eur J Pediatr 2009;168(5):515-22.

6. Barst RJ, Maislin G, Fishman AP. Vasodilator therapy for primary pulmonary hypertension in children. Circulation 1999;99(9):1197-208.

7. Roofthooft MT, van Loon RL, Berger RM. Management of pulmonary arterial hypertension in children. Paediatr Respir Rev 2010;11(4):240-5.

8. Patocka C, Nemeth J. Pulmonary embolism in pediatrics. J Emerg Med 2012;42(1):105-16.

9. Van Ommen $\mathrm{CH}$, Peters M. Acute pulmonary embolism in childhood. Thromb Res 2006;118(1):13-25.

10. Widlitz A, Barst RJ. Pulmonary arterial hypertension in children. Eur Respir J 2003;21(1):155-76.

11. Goldhaber SZ. Echocardiography in the management of pulmonary embolism. Ann Intern Med 2002;136(9):691-700.

12. Monagle $\mathrm{P}$, Adams M, Mahoney M, Ali K, et al. Outcome of pediatric thromboembolic disease: a report from the Canadian Childhood Thrombophilia Registry. Pediatr Res 2000;47(6):763-6. 